\title{
Time Frequency Analysis of Spacecraft Propellant Tank Spinning Slosh
}

\author{
Steven T. Green' and Russell C. Burkey² \\ Southwest Research Institute ${ }^{\otimes}$, San Antonio, Texas, 78238 \\ and \\ James Sudermann ${ }^{3}$ \\ NASA Kennedy Space Center, Florida, 32899
}

\begin{abstract}
Many spacecraft are designed to spin about an axis along the flight path as a means of stabilizing the attitude of the spacecraft via gyroscopic stiffness. Because of the assembly requirements of the spacecraft and the launch vehicle, these spacecraft often spin about an axis corresponding to a minor moment of inertia. In such a case, any perturbation of the spin axis will cause sloshing motions in the liquid propellant tanks that will eventually dissipate enough kinetic energy to cause the spin axis nutation (wobble) to grow further. This spinning slosh and resultant nutation growth is a primary design problem of spinning spacecraft and one that is not easily solved by analysis or simulation only. Testing remains the surest way to address spacecraft nutation growth. This paper describes a test method and data analysis technique that reveal the resonant frequency and damping behavior of liquid motions in a spinning tank. Slosh resonant frequency and damping characteristics are necessary inputs to any accurate numerical dynamic simulation of the spacecraft.
\end{abstract}

\section{Nomenclature}

$\Omega_{\text {spin }}=$ total spin rate

$M_{r}=$ radial moment

$\lambda \quad=$ nutation frequency

\section{Introduction}

$\mathrm{D}$ ESIGNERS of spacecraft that spin for stability and pointing purposes must take into account the peculiar dynamics associated with spinning. Of particular concern are the effects of energy dissipation on the total system. A system spinning about its major inertial axis, like a Frisbee, is in a "flat-spin" condition and is in a low energy state. A system spinning about a minor inertial axis, like a pencil spinning about its long axis, is at a high energy state. In the absence of other external forces, such as in the zero-g vacuum environment of space, any energy loss from the system will force the spin to translate from the higher energy minor axis to the lower energy "flatspin" about the major axis. This phenomena was seen in the very first US spacecraft, Explorer 1, when flex of the long whip antennas used to measure the Van Allen belt radiation caused the pencil-shaped spacecraft to transition, over a period of time, into a flat spin about its center of gravity. This tendency of ever-increasing wobble (nutation) can lead to many forms of undesirable behavior besides the ultimate flat spin. Cork screwing during propulsive burns, general loss of control, and excessive attitude and pointing errors have all been documented in missions throughout the history of space flight. While flexible structures are an important area of concern, the primary source of energy dissipation is liquid fuel sloshing about in spacecraft and launch vehicle fuel tanks. This fuel slosh and its effect on the spacecraft and launch vehicle dynamics must be analyzed and understood in order to assure mission success.

\footnotetext{
' Staff Engineer, Fluid Dynamics and Multiphase Flow, 6220 Culebra Road., AlAA Member.

${ }^{2}$ Senior Research Engineer, Fluid Dynamics and Multiphase Flow, 6220 Culebra Road., AIAA Non-Member.

${ }^{3}$ Controls Analyst, ELV Control Systems Analysis, Mail Code VA-F3, AIAA Member. 
In the analysis of dynamic systems, such as spinning and nutating spacecraft, it is often advantageous to model the system in a simplified manner so that parameters can be randomly varied over a reasonable range and statistics can be taken on a multitude of simulation cases. Such Monte Carlo methods are commonly used to quantify the probability of mission success and to identify and analyze areas of sensitivity and concern. In order to construct a reduced model that yields realistic results, but yet is simple enough to run thousands or tens of thousands of times, the various model parameters must be understood as accurately as possible and must be derived with a minimum of assumptions. Some type of testing of the physical systems involved is the preferred method for increasing the level of confidence in the model parameters. The fluid behavior inside the spacecraft fuel tanks is usually the least understood physical system and is, therefore, the most likely to benefit from some type of test.

One of the current test methods for nutation growth assessment is the drop test in which small-scale spacecraft models are spun and dropped to observe their spin behavior in the nearly $0 \mathrm{~g}$ during the free fall. This method directly measures the nutation of the falling spacecraft model, but requires great skill and experience to scale the combination of fluid and diaphragm dynamics and it does not reveal sufficient information to construct a predictive computer simulation. CFD codes are an accurate and useful method for fluid modeling, but unfortunately are too computationally intensive to generate the thousands of cases necessary for a Monte Carlo statistical analysis. Another current test method imposes a forced motion on full-scale spacecraft tank models. In this method, the fluid forces are analyzed with respect to the imposed nutation excitation to reveal the natural frequencies and effective damping of the fluid rotary slosh. This method does not use scale models, but nutation is not allowed to grow and the testing is, by necessity, done in a $1-g$ environment. Hubert ${ }^{1}$ describes the types of spinning slosh test facilities and presents a review of much of the test data obtained from the different laboratories.

\section{Test Setup}

The Spinning Slosh Test Rig (SSTR), Figure 1, is a facility for testing the fluid dynamic effects in full-scale models of spacecraft fuel tanks. The SSTR is operated by Southwest Research Institute (SwRI ${ }^{B}$ ) for NASA's Kennedy Space Center (KSC). The SSTR provides a forced motion environment that simulates the nutation of a spacecraft. The SSTR test data are typically used to develop mechanical analogs (e.g., pendulums and rotors) for fluid motions that are used in numerical spacecraft dynamics models and for estimating the energy dissipation in the fluid as it is related to the time constant for the growth of the spacecraft nutation cone angle. Figure 1 shows the SSTR with a tank mounted on the spin axis, but the test facility can also accommodate tanks mounted up to about $1.5 \mathrm{~m}$ off-axis. The original design and operation of the SSTR for off-axis and on-axis operations are described in detail by Green et al. ${ }^{2}$, and Green et al. ${ }^{3}$.

The SSTR produces the forced motion by varying the speeds of the two motors. The lower motor spins the tilted upper frame to which the upper motor is mounted. The upper motor spins the tank relative to the upper frame. The overall spin rate is essentially the sum of the two motor speeds (the $3.1^{\circ}$ cone angle introduces a small correction factor to this relationship). The simulated nutation amplitude is proportional to the lower motor spin rate and the simulated nutation frequency is proportional to the upper motor spin rate. The six load components (forces and moments) on the test tank are measured using three triaxial dynamic force sensors contained within the dynamometer that rigidly attaches the tank to the wobbling platform.

Historically, SSTR testing has been conducted by measuring the fluid loads at a large matrix of fixed motor speeds over a

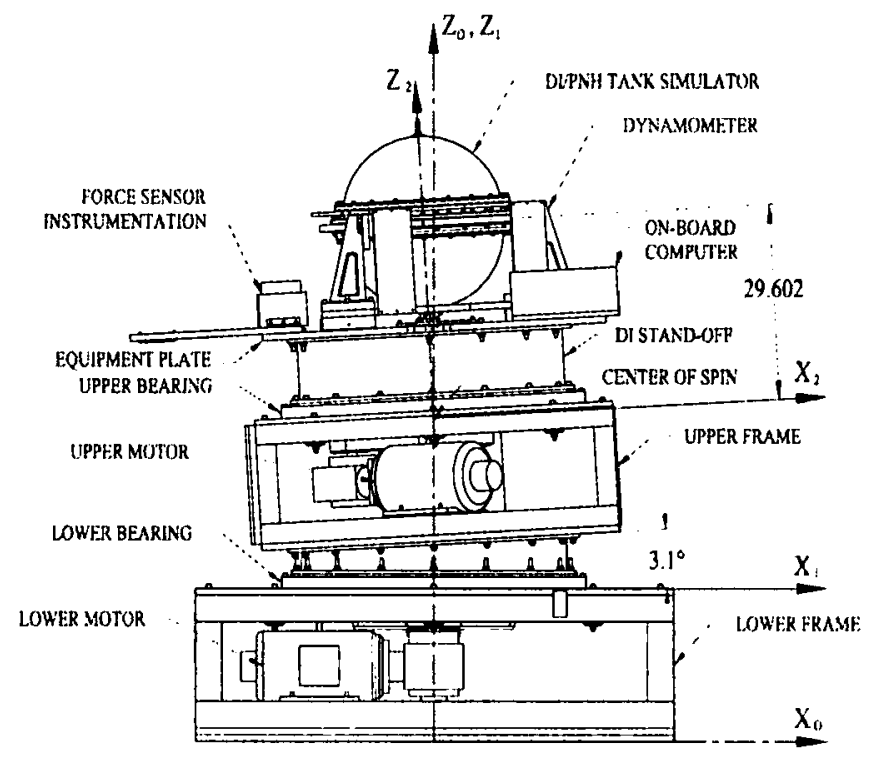

Figure 1. Slosh Test Rig (SSTR). 
range of nutation frequencies and spin rates. The amplitude and phase relations of the loads with respect to the imposed kinematic conditions are analyzed to reveal the dynamic characteristics of these loads and allow the specification of mechanical analogs that mimic the resonance and damping behavior of the fluid loads on the tank. This process is tedious and the data quality is not apparent until the lengthy post-processing is completed.

The objective of the testing described here was to investigate test procedures and the corresponding data analysis methods that could speed the entire data acquisition and mechanical model selection processes for the ultimate needs of the overall spacecraft dynamics analyses. The tests described here are from a series of experiments performed with a full-scale tank model that is geometrically similar to the one used in the Deep Impact and Pluto New Horizons (DI/PNH) spacecraft. The tests do not, however, correspond to any specific spacecraft mission.

The DI/PNH spacecraft propellant tank is a nominal 23-inch internal diameter cylindrospherical tank with a diaphragm fixed near the tank equator. In flight, the tank is oriented such that the diaphragm is forward of the liquid when the upper-stage rocket is firing. In testing, the tank simulator is oriented such that the diaphragm is above the liquid.

The DI/PNH tank simulator, shown in Figure 2, has acrylic hemispherical domes with a twopiece aluminum center cylindrical section. The tank simulator acrylic dome walls are 0.5 inches thick and fill and vent ports are centrally located in each hemisphere. The aluminum diaphragm retaining rings clamp the diaphragm and provide for attachment to the dynamometer assembly. The diaphragm clamp rings can be replaced with tank rings that have no diaphragm attachment features so that tests can be conducted on a cylindrospherical, diaphragmless tank simulator. The tank simulator itself weighs $82 \mathrm{lb}$ and holds $269 \mathrm{lb}$ of water at $100 \%$ full.

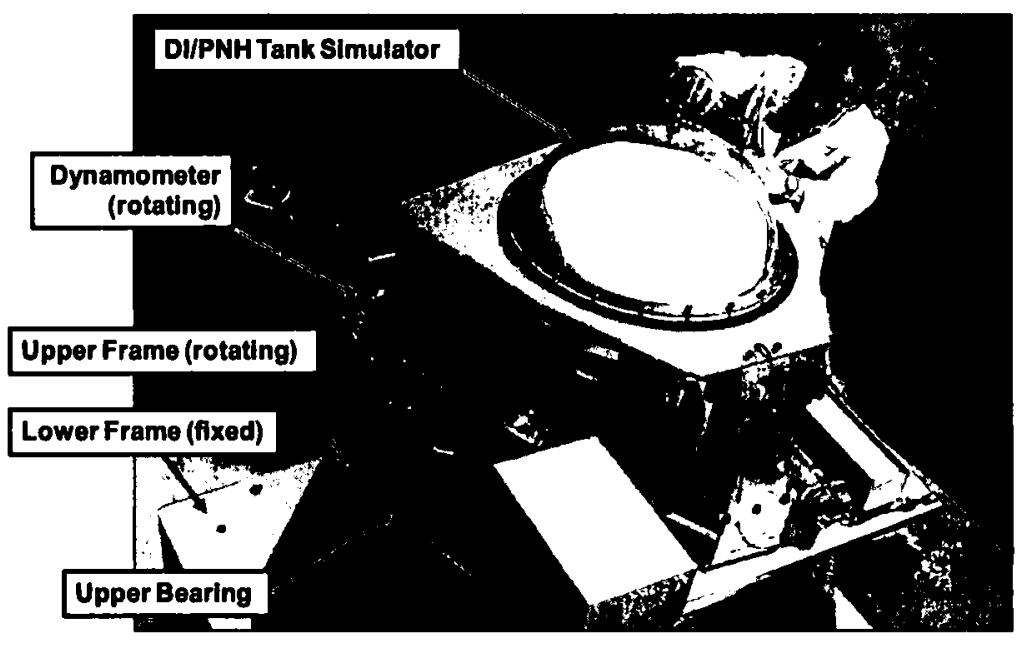

Figure 2. DI/PNH Tank Simulator.

The tank simulator diaphragm may be manipulated into a variety of shapes. The diaphragm shapes that have been tested (crater, mountain, yin-yang, and ridge) are sketched in Figure 3. Only the crater and mountain shapes were used in the tests performed here
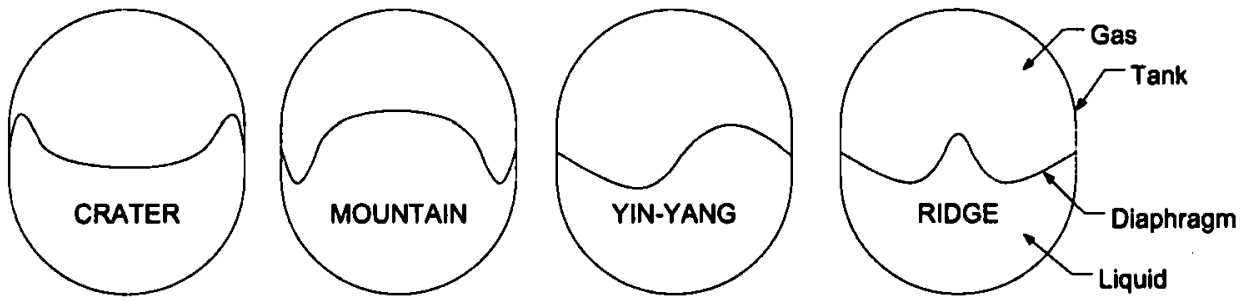

Figure 3. Diaphragm Shapes.

\section{Test Matrix}

Tests were conducted in this program using the diaphragm tank configuration and the tank without a diaphragm (i.e., termed "bare"); although, the diaphragm configuration is much more commonly used in spacecraft. The motivation for including the bare tank in the test program is that previous testing (Green et al ${ }^{4}$ ) has shown there is a 
strong, clear, lightly-damped resonance observable in the fluid moment loads on the tank. This is contrasted against the diaphragm tank in which previous testing of on-axis tanks (Green et al. ${ }^{4}$ ) has shown that the resonance observed in the fluid torques is highly damped and fairly difficult to detect. Thus, the revised test methodology and data analysis processes described in this paper are applied to situations where resonances are known to exist - one in which the resonance is easy to detect (i.e., the bare tank) and one in which the resonance is difficult to detect (i.e., the diaphragm tank).

Furthermore, the resonances in previous bare tank testing have been observed as amplification and a large phase shift in the moment loads at a nutation frequency of $90 \%$ to $95 \%$ of the spin rate, which is just at the largest nutation frequency previously tested. Therefore, for the bare tank testing, the nutation frequency range (as a percent of total spin rate) was extended to $120 \%$ of spin rate, in order to have data at nutation frequencies greater than the resonant frequency. This was accomplished by spinning the upper motor faster than $120 \mathrm{rpm}$, while simultaneously spinning the lower motor backwards and producing retrograde motion.

Two of the basic test protocols used in the testing program are described here. First, a classical sweep of nutation frequencies at a constant spin rate was conducted for each tank fill level. This was accomplished by coordinating the speeds of the upper and lower motors in the SSTR to provide for a prescribed sweep of the nutation frequency (i.e., upper motor speed) while the sum of the upper and lower motor speeds is constant. This type of test is called the "Nutation Sweep" in the nomenclature of this test program. Second, the loads on the tank in response to a rapid change in nutation frequency at a constant spin rate were measured. This was accomplished by only spinning the tank with the lower motor and then, changing the upper and lower motor speeds as quickly as possible to a final combination where a specific nutation frequency was achieved at the original overall spin rate. This type of test is called the "Spin Only Plus Nutation" in the nomenclature of this test program. This second test method is akin to a 'bump' test of a structural system response. The test matrices in Table 1 and Table 2 summarize only those tests that are discussed in this paper. The test program used many other combinations of speed changes, but only those tests with notable features are discussed here.

Table 1. Bare Tank Configuration Test Matrix

\begin{tabular}{|c|c|c|c|c|c|}
\hline Test & $\begin{array}{c}\text { Total Spin } \\
\text { Rate (rpm) }\end{array}$ & $\begin{array}{c}\text { Nutation } \\
\text { Rate } \\
\text { (\% of Total } \\
\text { Spin Rate) }\end{array}$ & $\begin{array}{c}\text { Upper } \\
\text { Motor } \\
\text { (rpm) }\end{array}$ & $\begin{array}{c}\text { Lower } \\
\text { Motor } \\
\text { (rpm) }\end{array}$ & $\begin{array}{c}\text { Fill Levels } \\
\text { \% Volume }\end{array}$ \\
\hline $\begin{array}{c}\text { Nutation } \\
\text { Sweep }\end{array}$ & 120 & 50 to 120 & 60 to 144 & 60 to -24 & 62.4 and 100 \\
\hline $\begin{array}{c}\text { Spin Only } \\
\text { Plus } \\
\text { Nutation }\end{array}$ & 120 & 0 to 120 & 0 to 144 & 120 to -24 & 62.4 and 100 \\
\hline
\end{tabular}

1. Nutation Sweep Tests used an exponential sweep over six minutes.

2. Spin Only Plus Nutation Tests included final nutation rates of $20 \%, 50 \%, 80 \%$, and $120 \%$ of total spin rate.

Table 2. Diaphragm Tank Configuration Test Matrix

\begin{tabular}{|c|c|c|c|c|c|}
\hline Test & $\begin{array}{c}\text { Total Spin } \\
\text { Rate (rpm) }\end{array}$ & $\begin{array}{c}\text { Nutation } \\
\text { Rate } \\
\text { (\% of Total } \\
\text { Spin Rate) }\end{array}$ & $\begin{array}{c}\text { Upper } \\
\text { Motor } \\
\text { (rpm) }\end{array}$ & $\begin{array}{c}\text { Lower } \\
\text { Motor } \\
\text { (rpm) }\end{array}$ & $\begin{array}{c}\text { Fill Levels } \\
\text { \% Volume }\end{array}$ \\
\hline $\begin{array}{c}\text { Nutation } \\
\text { Sweep }\end{array}$ & 60 & 10 to 95 & 6 to 57 & 54 to 3 & 62.4 and 80 \\
\hline $\begin{array}{c}\text { Spin Only } \\
\text { Plus } \\
\text { Nutation }\end{array}$ & 60 & 0 to 80 & 0 to 48 & 60 to 12 & 62.4 and 80 \\
\hline
\end{tabular}

1. Nutation Sweep Tests used an exponential sweep over six minutes.

2. Spin Only Plus Nutation Tests included nutation rates of $20 \%, 50 \%$, and $80 \%$ of total spin rate.

4

American Institute of Aeronautics and Astronautics 


\section{Test Results}

The time histories of the SSTR force sensors are processed to obtain the six components of the loads experienced by the tank as the SSTR operates. For the sake of brevity, the details of the data processing are not included here, but are fully described by Green et al. ${ }^{3}$. The time-frequency analysis of the spinning slosh test results are presented in this section. All of the analyses were performed within the MATLAB ${ }^{5}$ software suite. The results of the nutation sweep tests are described first and are followed by a description of the rapid nutation change test.

\section{A. Nutation Sweep Tests}

The total spin rate, $\Omega_{\text {spin }}$, was held constant at $120 \mathrm{rpm}$ in the bare tank tests. This provides an acceleration of about $4.8 \mathrm{~g}$ at the outermost tank wall and, in the case of a tank filled to less than $100 \%$, produces a deep parabolic trough in the fluid surface that approaches the total fluid surface area that would be produced in $0 \mathrm{~g}$. The total spin rate in the diaphragm tank tests was $60 \mathrm{rpm}$. This is similar to the spin rate of the launch vehicle upper stage.

\section{Bare Tank, 100\% Fill Level}

The nutation sweep test for the $100 \%$ fill level bare tank was conducted at a total spin rate $\left(\Omega_{\text {spin }}\right)$ of $120 \mathrm{rpm}$ and covered a nutation frequency range, $\lambda$, of $60 \mathrm{rpm}$ to $144 \mathrm{rpm}\left(\lambda=0.5 \Omega_{\text {spin }}\right.$ to $\left.1.2 \Omega_{\text {spin }}\right)$. The sweep followed an exponential profile over about a sixminute period. The spectrogram of the measured lateral moment, $M_{r}$, for this test is shown in Figure 4. The graphics in Figure 4 are typical of the other spectrograms in this paper. It is a composite of the time history of the signal being observed and the spectrogram of the signal. The colors in the spectrogram are scaled to the magnitude of the complex Fourier transform of the input signal and are in the logarithmic units of $\mathrm{dB}$ to enhance the lower amplitude values.

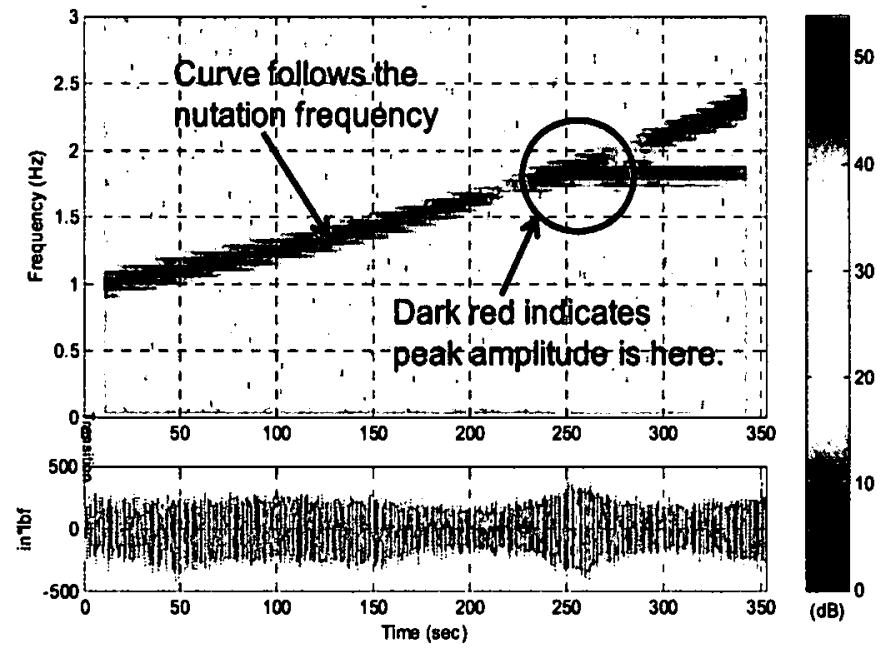

Figure 4. Moment Load Spectrogram for Bare Tank, $100 \%$ Fill Level, Nutation Sweep.

The red curve beginning at a frequency of $1 \mathrm{~Hz}$ at the left of the graph shows the magnitude of the moment signal at the nutation frequency. This curve slowly decreases in color intensity, indicating a decrease in the magnitude of the peak moment signal until the frequency reaches about $1.9 \mathrm{~Hz}=0.95 \Omega_{\text {spin. }}$. At that point, there is a rapid increase in the moment amplitude. Visual observation of neutrally-buoyant marker particles in the tank confirms resonance behavior at this frequency. For frequencies greater than $1.9 \mathrm{~Hz}$, there are two local maxima in the moment amplitude as a function of frequency. A graph of the amplitude along this red curve (constructed by selecting the maximum spectrogram value at each point along the time axis) is shown in Figure 5. It should be noted that the ripples in the graph of Figure 3 are an artifact of the windowing specified for the Fourier transforms used in producing the spectrogram values.

One branch of the bifurcation in Figure 4 remains at constant frequency for the remainder of the test, while the other continues to increase in frequency along with the nutation frequency. The lower constant frequency branch indicates that the resonance at $1.9 \mathrm{~Hz}$ is still being excited past the time when the nutation frequency has passed through this value. 
It can be shown that the excitations for the lateral slosh moments are proportional to the bodycentered rotation rates orthogonal to the respective moment. With this in mind, the transfer function for the radial moment is shown in Figure 6 for the time at which the nutation frequency is $0.95 \Omega_{\text {spin }}$ $=1.9 \mathrm{~Hz}$. The phase plots also show a deviation from the respective rigid body values of about $120^{\circ}$. Note also that the low frequency range of the phase plot is in agreement with the rigid body assumption.

\section{Bare Tank, 62\% Fill Level}

The bare tank at $62 \%$ fill level was subjected to the same nutation sweep test as in the case of the $100 \%$ fill level tank. The spectrogram of the $62 \%$ fill level nutation sweep test is shown in Figure 7. As in the case of the full tank, the dominant signal in the spectrogram follows the nutation frequency and gradually decays in amplitude until a frequency of $2.1 \mathrm{~Hz}\left(\lambda=1.05 \Omega_{\text {spin }}\right)$ is reached where there is a local maximum in the amplitude. After passing through the local maximum, the amplitude again slowly increases. Unlike the $100 \%$ fill level case, there is no sustained signal at the resonance frequency after the nutation sweep passes through the resonance value.

The variation of the peak $M_{r}$ signal amplitude with nutation frequency for the $62 \%$ fill level case is displayed in Figure 8. The value of $M_{r}$ at the low end of the frequency range is similar to that of the $100 \%$ fill level case. The center of mass for the $62 \%$ fill level case is further below the center of the tank than in the $100 \%$ fill level case; so, even though the mass is $38 \%$ less, the increased center of gravity offset results in moments for the rigid body mode to be similar for the two fill levels. The primary differences in the moments for the two fill levels is that in the $62 \%$ fill level case the resonance frequency is slightly greater, but the resonance amplitude is only $30 \%$ of the value for the $100 \%$ fill level case.

\section{Diaphragm Tank (Crater Shape), 80\% Fill Level}

The tank with a crater-shaped diaphragm at a fill level of $80 \%$ was subjected to the same nutation sweep and rapid nutation change tests as for the bare tank. In the case of the diaphragm tank, however, the time-frequency analysis did not reveal the subtle resonance features that were observed from the previous steady nutation tests (see Green et al. ${ }^{3}$ ). For example, the spectrogram for the radial lateral moment measured during the

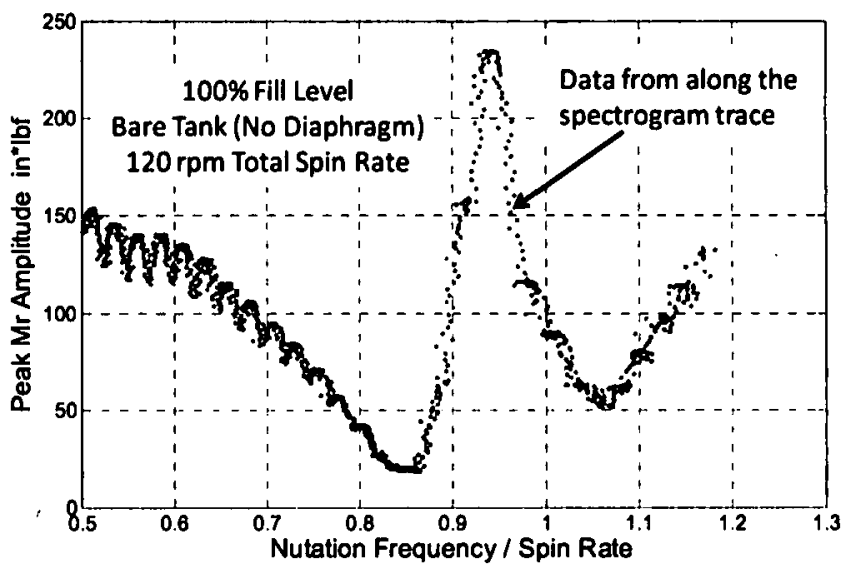

Figure 5. Peak Spectrogram Values for Moment Load, Bare Tank, $\mathbf{1 0 0 \%}$ Fill Level, Nutation Sweep.

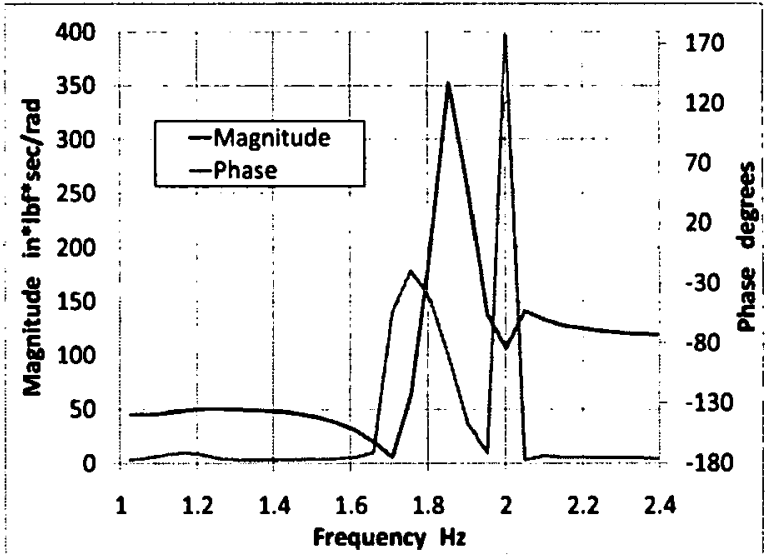

Figure 6. Radial Moment Transfer Function for Bare Tank, $100 \%$ Fill Level, Nutation Sweep.

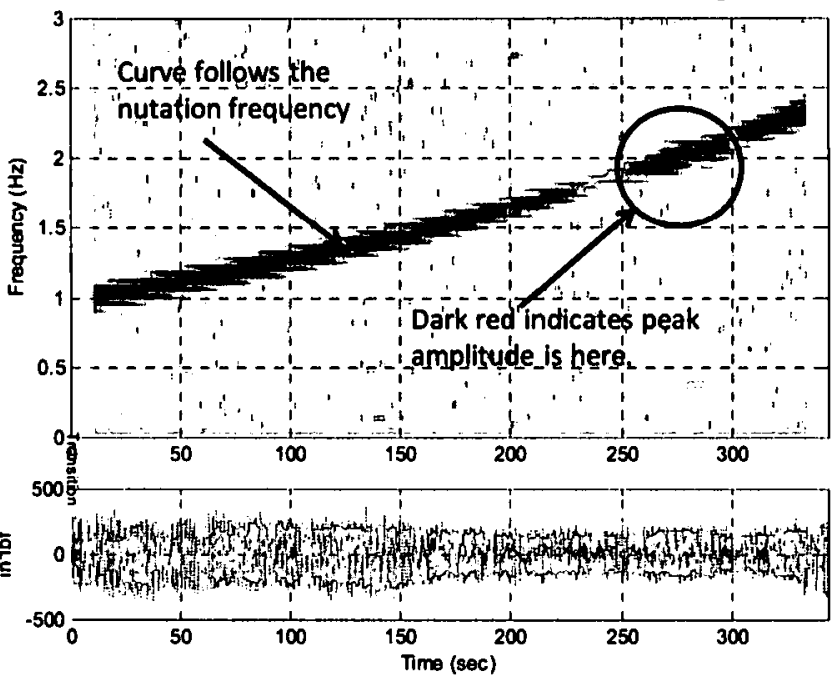

Figure 7. Moment Load Spectrogram for Bare Tank, $62 \%$ Fill Level, Nutation Sweep.

6

American Institute of Aeronautics and Astronautics 
nutation sweep test is shown in Figure 9. There are second and third order harmonics of the base excitation frequency clearly present. There is, however, no discernible resonance feature, even though the steady nutation tests show that there is a subtle resonance for this fill level at about $0.65 \Omega_{\text {spin }}$. It was thought that the damping imparted by the diaphragm masks the resonance in the sweep tests to a greater degree than in the steady nutation tests. Even for sweep profile durations of 26 minutes (compared to the six-minute durations used in all other tests), however, the resonance in the fluid loads was not detected here.

The variation of the peak radial moment signal amplitude with nutation frequency for the diaphragm tank with a crater shape for the $80 \%$ fill level case is displayed in Figure 10. As in the full spectrogram, there is not a recognizable resonance feature in this graph. Indeed, discounting the ripples in the plot, the curve is similar in shape and magnitude to that expected for a rigid body.

The $62 \%$ fill level crater and mountain shape diaphragm tests yielded similar results and conclusions in comparison to the $80 \%$ fill level crater shape diaphragm tests. No results from these tests are given here.

\section{B. Rapid Nutation Change Test}

This test was envisioned to be analogous to an impact load in a structural response test, except in this case, a "step" input is applied in order to excite a broad range of frequencies in an attempt to observe resonance features. Several versions of this test were attempted with the most enlightening being the rapid application of nutation after a steady spinonly initial condition. The nutation frequency step change ranged from $25 \%$ of $\Omega_{\text {spin }}$ to $120 \%$ of $\Omega_{\text {spin }}$, but in all cases shown here, the initial condition of the test was at no nutation (i.e., upper motor not active).

\section{Bare Tank, 100\% Fill Level}

The spectrogram for the test in which the step change in nutation frequency was to $96 \mathrm{rpm}$ $\left(0.8 \Omega_{\text {spin }}\right)$ is shown in Figure 11 . After the completion of the step change in nutation, the dominate trace in the spectrogram is due to the nutation frequency of $1.6 \mathrm{~Hz}(96 \mathrm{rpm})$ main trace. There is a secondary trace at the resonance frequency of about $1.9 \mathrm{~Hz}$, which decays within about 60 seconds after the final nutation frequency is reached. This excitation of the resonance is caused even though the system did not intentionally pass

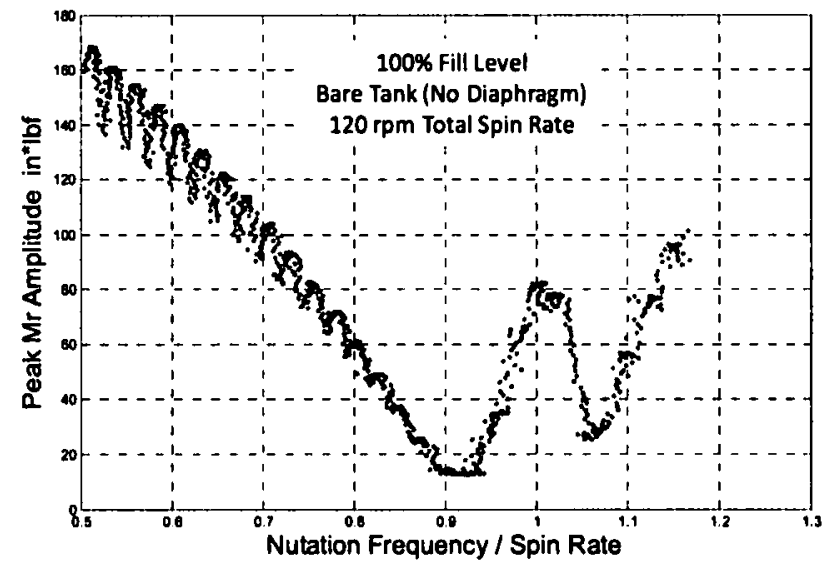

Figure 8. Peak Spectrogram Values for Moment Load, Bare Tank, 62\% Fill Level, Nutation Sweep.

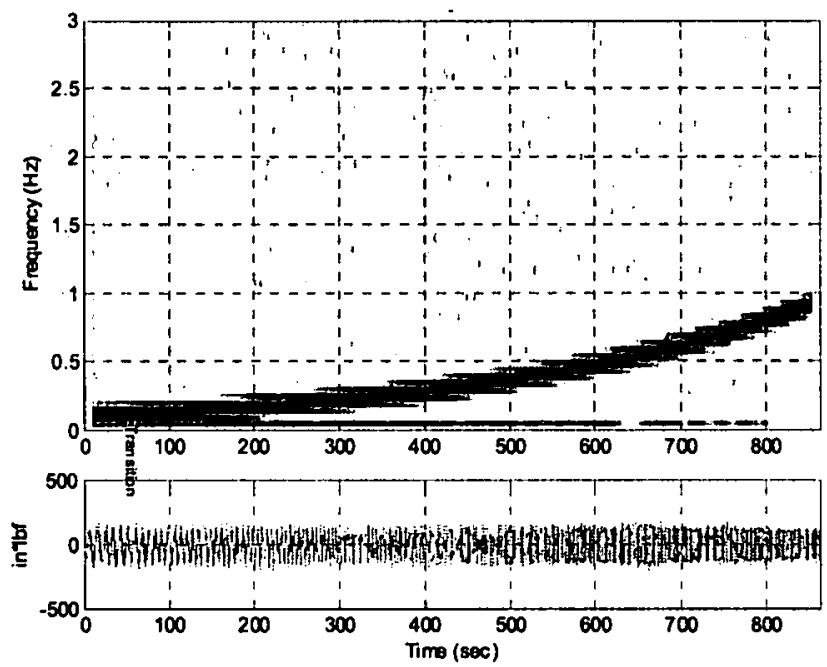

Figure 9. Moment Load Spectrogram for Crater Diaphragm Tank, 80\% Fill Level, Nutation Sweep.

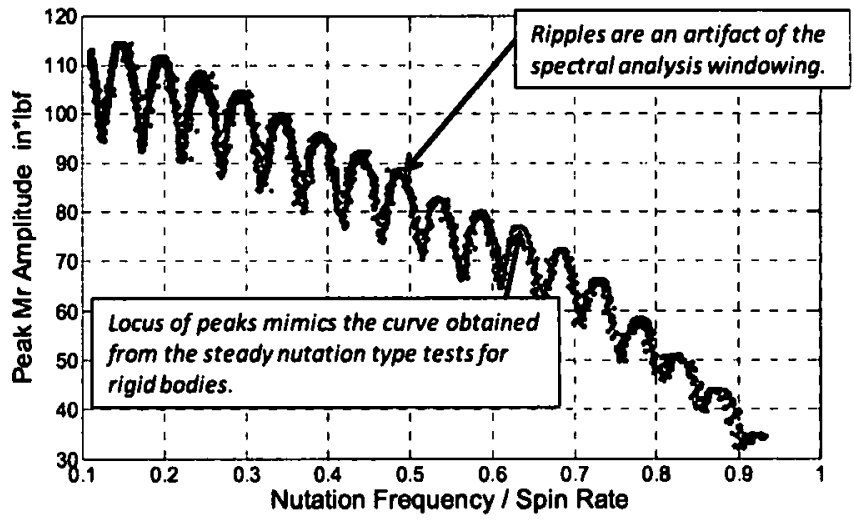

Figure 10. Peak Spectrogram Values for Radial Moment Load, Crater Diaphragm Tank, 80\% Fill Level, Nutation Sweep.

American Institute of Aeronautics and Astronautics 
through the resonance frequency. This is, of course, as expected from the step input to the imposed motion.

Similarly, the spectrogram for the test in which the step change in nutation frequency was to $144 \mathrm{rpm}$ (1.2 $\Omega_{\text {spin }}$ ), is shown in Figure 12. The dominant excitation frequency is $25 \%$ greater than the resonance frequency, but resonance motion was excited during the step change. The resonance portion of the signal is present for over 140 seconds. This decay is longer than in the case of a step input to $1.2 \Omega_{\text {spin }}$ duration, because the imposed motion explicitly included the resonance frequency.

\section{Bare Tank, 62\% Fill Level}

The results of the most extreme of the tests for transition from spin only to nutation for the $62 \%$ fill level tank are shown in Figure 13. The final nutation frequency is about $20 \%$ greater than the resonance frequency, but the signal at the resonance frequency is excited for a short time. This signal decays in about 20 seconds, which is much sooner than in the corresponding test for the $100 \%$ fill level case. The presence of a fluid surface clearly alters the amplification and damping characteristics of the resonance.

\section{Conclusions}

Previously, the apprdach to using the SSTR test facility was to gather data from several tests that were conducted at steady nutation frequencies. The parameters of a mechanical analog consisting of a pendulum and rotors (e.g., pendulum mass and length, diaphragm stiffness and damping, rotor inertia and stiffness, etc.) were then estimated by comparing the dynamic response of the mechanical system to the measured response of the test tank. The estimation process was accomplished either by the fitting of the linearized equations of motion for the mechanical analog or a computational equivalent of the mechanical system in a modeling package such as MATLAB/Simulink. Either method was tedious and required the handling of many different data sets.

Several alternative approaches to the SSTR configuration, testing parameters, and data analysis techniques were implemented and demonstrated in this project. As demonstrated here, the testing techniques that show promise of streamlining the slosh model parameter estimation are the nutation sweep and the transition from spin only to nutation. These test techniques, coupled with a time-frequency analysis will provide a single data set (or at least fewer data sets) as the basis of the parameter fitting procedure. The ultimate goal of all of the analyses is to provide a

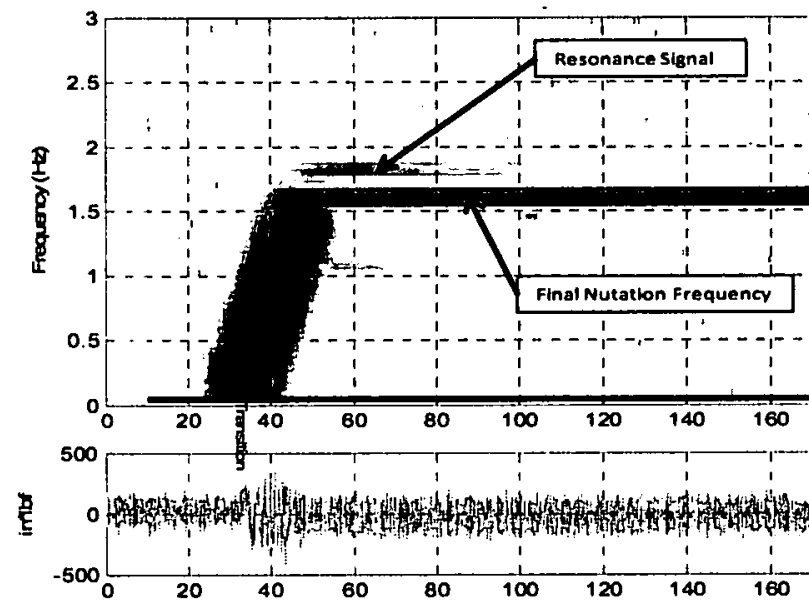

Figure 11. Moment Load Spectrogram for Bare Tank, $100 \%$ Fill Level, Spin Only Plus Nutation at $0.8 \boldsymbol{\Omega}_{\text {spin }}$.

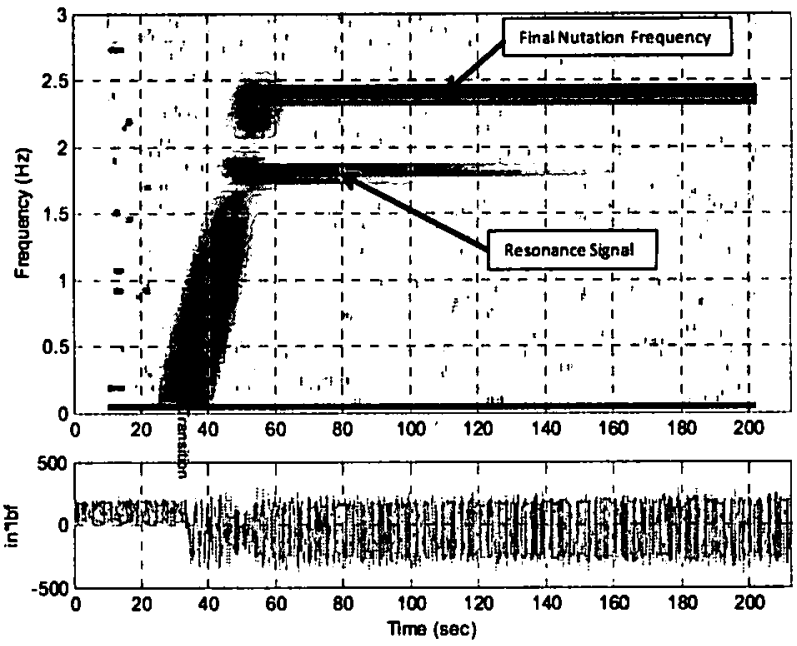

Figure 12. Moment Load Spectrogram for Bare Tank, $100 \%$ Fill Level, Spin Only Plus Nutation at $1.2 \Omega_{\text {spin }}$.

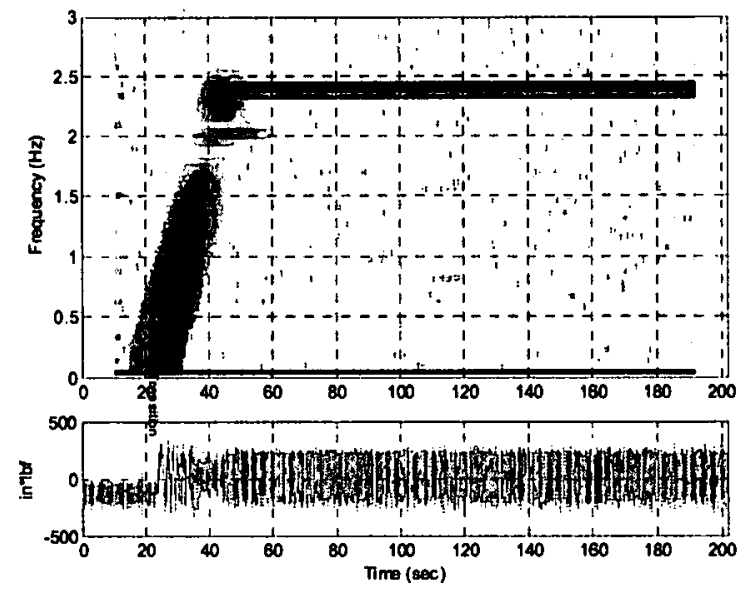

Figure 13. Moment Load Spectrogram for Bare Tank, $62 \%$ Fill Level, Spin Only Plus Nutation at $1.2 \Omega_{\text {spin }}$. 8

American Institute of Aeronautics and Astronautics 
reasonable estimate for the nutation time constant of the spacecraft. The nutation time constant is typically at its minimum if the propellant in the tanks is excited at its slosh resonance where it provides for the worst case of spacecraft energy dissipation.

These alternative approaches will work well for bare tanks where the resonance features are clearly isolated as demonstrated here. Unfortunately, the procedures explored here are not as useful in determining the presence of resonance motions for the diaphragm tanks tested here. It should be remembered, however, that in the tests conducted here, the tank was located on the simulated spin axis of the spacecraft. The driving force for excitation of spinning slosh at this position is much less than for tanks located off the spin axis (e.g., at the spacecraft periphery).

Based on the success of the alternative test methods and data analysis processes, the recommendations to extend this work are as follows:

- Investigate the use of nutation sweep tests, rapid nutation change tests, and spin only plus nutation tests for offaxis tanks. These off-axis tests should include both diaphragm tanks and bare tanks.

- Extend the time-frequency analysis methods to include the estimation of mechanical analog (pendulum and rotor) model parameters for slosh dynamics.

- Incorporate the time-frequency data analysis methods and mechanical analog model parameter estimation into a complete system within the MATLAB/Simulink framework.

\section{Acknowledgments}

The work described in this paper was done as part of Southwest Research Institute Project 18.14208 under contract number NNK08LB12C with NASA Kennedy Space Center. The composite views of a spectrogram with the time history were produced by a special MATLAB function supplied by Dave Griffin of NASA-KSC.

\section{References}

'Hubert, C., "Behavior of Spinning Space Vehicles with Onboard Liquids," Second Edition, Final Report, NASA Contract NNK05LA48P, 2008.

${ }^{2}$ Green, S. T., Wattis, G. N., Burkey, R. C., Walter, D. B., Dodge, F. T., and Kuhl, C. A., "Spinning Slosh Test Rig: Hardware Design and Operating Procedures," Final Report, Vol. 2, Boeing Contract K59000001, SwRI Project $18.03513,2000$.

${ }^{3}$ Green, S. T., Burkey, R. C., "Nutation Time Constant Model Parameters for the SSTR On-Axis Configuration (Deep Impact/Pluto New Horizons Spacecraft) - Task 2 Report," NASA Contract No. NNK08LB12C, SwRI Project 18.14208, 2009.

${ }^{4}$ Green, S. T., Burkey, R. C., Walter, D. B., "Nutation Time Constant Model Parameters for the SSTR On-Axis Configuration (Deep Impact/Pluto New Horizons Spacecraft) - Final Report," NASA Contract No. NNK06LA44P, SwRI Project 18.12441, 2007.

${ }^{5}$ MATLAB, Version 2008b, The MathWorks, Inc., Natick, Massachusetts. 